\title{
Incorporating the iPad2 in the Mathematics Classroom: Extending the Mind into the Collective
}

\author{
http://dx.doi.org/10.3991/ijep.v2i2.2084 \\ P. Preciado-Babb \\ Galileo Educational Network and University of Calgary, Calgary, Canada
}

\begin{abstract}
Doubtlessly, mathematics is one of the most important subjects in education from $\mathrm{K}$ to 12 levels especially for students interested in eventually pursuing undergraduate studies in the fields of science and technology. As it has been argued in mathematics education research, not only the content, but also the form in which students learn is important for mathematics learning. Particularly, an inquiry approach permeates through the mathematics curriculum of several countries around the world. Additionally, the use of technology to learn mathematics has been increasing in the last decades, requiring teachers and professionals in education to constantly explore and learn new possibilities or affordances in the classroom. The purpose of this paper is to initiate a discussion about the possible and complex forms of interaction among students, teacher, mathematical tasks, and the electronic tablet (iPad2) in an inquiry learning environment. An experience from a grade 10 classroom is used as a context to exemplify these interactions.
\end{abstract}

Index Terms-Embodied cognition, iPad, Mathematics, Problem solving, Technology.

\section{INTRODUCTION}

This article is an extended version of a paper submitted to the third IEEE Global Education Conference, 2012. The experience of the use of the iPad in the classroom described in the original submission is elaborated with more details in this article. Additional evidence of students' work is presented as well.

The use of technology in the mathematics classroom within inquiry based learning environments has increased significantly in the past few decades. Research on mathematics education regarding the use of technology for teaching and learning is extensive, as demonstrated by findings in the many specialized journals and conferences held internationally. For instance, the international group of the Psychology of the Mathematics Education (PME) has focused on cognitive aspects of learning and teaching mathematics with technology (e.g. [1]), particularly in the use of the computer and the graphic calculator for learning algebra, calculus and geometry. However, newer technologies, inclusive of mobile phones and electronic tablets such as the iPad, are now available and evident in the daily life of technological societies, representing new possibilities for the classroom.

An inquiry approach to the learning of mathematics requires student to engage actively in a variety of activities in the classroom different from those used in more teacher centered approaches. Terms such as 'problem solving' and 'mathematics reasoning' are present in much of the curriculum of several countries (e.g. [2], [3], [4], and [5]). The mathematics programs of several provinces in Canada incorporate critical components for learning comprising the following processes: communication, connections, mental mathematics and estimation, problem solving, technology, and visualization ([2] and [3]). An important part of the inquiry approach is that students are expected to learning through problem solving, as opposed to learn for problem solving. For instance, the Western and Northern Canadian Protocol for collaboration in education (WNCP) stresses that "if students have already been given ways to solve the problem, it is not a problem, but practice" ([2], p. 8). Mathematics problems must be understood as learning tasks for students who do not know how to solve them in advance. Instead of showing students a method to solve a type of mathematical tasks, teachers use students' generated solutions and approaches to enact mathematical concepts in the classroom. Moreover, teachers could learn from their students' novel strategies to solve mathematics problems, (e.g. [6]). Such strategies may include the use of a particular tool in a particular fashion.

Allowing students to use technological tools, such as the electronic tablet, for mathematical problem solving might represent a challenge for the teacher. Currently, technology is changing constantly and at high rate. As soon as we begin to master the use of a particular technology, new technologies soon become available with more and improved features. For instance, when preparing this article the new version of the iPad was on sale in March 2012, one year after the previous version, iPad 2. This 'innovation curve' represents a challenge for teachers interested in the incorporation of new technology in their classrooms, including "gaining knowledge and skills despite limited professional development” ([7], p. 1300). As new technologies, included the electronic tablet, are constantly introduced in the market, they become more accessible to anyone. People who were born and grew up using digital technology have been called 'Digital Natives' ([8]). As "most young people in many societies around the world carry mobile devices-cell phones, Sidekicks, iPones_at all times” ([8], p. 3), it becomes increasingly possible that many students have more experience with the new technologies than their teachers, particularly at the high school level ([9]). Because of this, today's teachers in many schools have ever-increasing opportunities to learn from their students as they generate ideas of how to incorporate these technologies in the classroom. While the 
PAPER

INCORPORATING THE IPAD2 IN THE MATHEMATICS CLASSROOM: EXTENDING THE MiND INTO THE COLLECTIVE

perspective of the teacher as a learner has been extended within the discipline of mathematics education (e.g. [6]), the students' potential contribution to the use of technology for mathematical problem solving is less documented. Such contribution not only represents new forms of interaction between teacher and student, but also new relationships among the mathematical tasks, including the intended mathematical content to be learned by the student, and the use of technology.

In this paper I considered the relationship between people and objects as both dynamic and reciprocal in order to explore the complex forms of interactions and relationships among students, teachers, mathematical tasks, and the iPad2, including its applications, in an inquiry learning environment at the high school level. For this purpose, I took an enactivist approach, which has its roots in the embodied cognition theory developed from neurscience ([10]), as well as some notions from neuroarchaeology ([11]). This approach allows to conceptualize both the technological tools, such as the iPad and its applications, and the mathematical task as evolving cultural entities that change as students, and teacher, learn more about, and find new forms of interaction with, them. For instance, the electronic tablet might be conceived only as a media reproduction and communication device for one student before using it in the classroom. However, after incorporating it as a tool for learning, it could represent something else to the student. In a similar way, a mathematics task may be perceived differently by a student, or even the teacher, when it has been solved or revisited from different perspectives.

In order to exemplify some of the ideas discussed here, I will reference my experience as mathematics educator working in the collaborative design of rich mathematical tasks in a public high school located in a low income sector of a city in Western Canada. While my descriptions of this experience would not count as research data, they provide a means to situate the discussions of this paper within a particular context.

\section{ENACTIVISM AND NEUROARCHAEOLOGY}

A number of contributions from neuroscience to cognitive learning theories are based on a framework of embodied cognition. In particular, the enactive theory of learning proposed by Varela and Maturana in [10] served to conceive and extend human cognition as embodied in the brain, as opposed to the Cartesian perspective that considers the mind separated from the body. More recently, neuroarchaeology has brought insights about how humans relate to objects in action within a cultural context, as explained in [11]. These two frameworks are explained in this section.

\section{A. Embodied Cognition}

Learning theories of representation, such as behaviorism, assume that individuals make representations of the outside world in their minds (see for example [10] and [12]). In these theories "knowledge is based on acquiring or picking up the relevant features of a pre-given world that can naturally decomposed into significant fragments" ([10] p. 253). This perception has been criticized so that alternative theories have been proposed that individuals make sense of their own experience with the environment as they interact with it. Maturana and Varela, based on biological and neurological studies, defined knowledge in terms of the sense that the individual makes to the world as he or she experiences it. Moreover, they made a phenomenological claim by arguing that "the knowledge of knowledge compels. ... It compels us to realize that the world everyone sees is not the world but $a$ world which we bring forth with others” ([10], p. 245, italics in original). Environment and organisms, human beings for example, experience a mutual story of co-evolution and transformation: This process makes different living organisms adapted and compatible with each other in the process of structural coupling. If we consider other individuals, as well as their artifacts in the environment, we also take into account social interactions, including the developed common history and culture as critical features in an individual's world. An important aspect within enactivism theory is that changes in the individual, or an organism, are triggered, but not determined by interaction with the environment or other individuals. Indeed, it is the individual's neuronal-body structure that allows these changes to occur. This process of change by such interactions is called structural determinism. In the case of student learning processes within a classroom, "the response to a stimulus is not in the stimulus per se but is in the person that responds to it. And in return, the teaching/learning situation is seen in a structural coupling sense, where a history of adapted meanings is being developed and to which both teacher and student are adapting and transforming, while keeping adapted” ([13], p.250).

According to [10], the story of co-evolution of organisms and environment is not determined by a process of natural selection based on efficiency. Rather, species evolve and diversify on a base of sufficiency. They call this the natural drift, premised upon the processes of structural coupling and structural determinism that emerge over long periods of time. However, in the local context of a classroom, it is possible to identify diversity in the actions and approaches that take place during class in which these two process are also involved: While structural determinism serves to understand that every student make his or her own sense of the learning environment, structural coupling entails the mutual adaptations of students, teacher, and environment. That is, there is a natural drift of interactions within the classroom.

As many individuals interact together in the processes of structural coupling and structural determinism, new complex entities are formed. For example, groups of people in an association or corporation are capable of undertaking endeavors that could not be possible by many individuals acting in isolation to each other. Taking into account these more complex entities as units, it make sense to talk about collective learning within a distributed network ([12] and [14]). This concept has been applied not only to human beings, but also in other learning systems such as networks in artificial intelligence settings. For instance, frameworks for intelligent actors designed to support learners have been proposed recently in engineering education (see [14]). Another example of a distributed teaching and learning network in education is the mathematics classroom where students are expected to participate by suggesting different approaches to mathematical tasks. The teacher is not the central source of knowledge: students also contribute to the development of the knowledge in the classroom. 
In the search of an understanding of the biodirectional links between brains, minds, and culture, neuroarchaeology has emerged as a set of ideas based on the notions of embodied cognition (see [11]). The relations between individuals and things in a cultural context are examined closely in this new field of study. The capacity of action of a human being depends on the form he or she uses or finds utility in specific things, which are embedded in a cultural context. In the relationship subject-action-thing, agency and intentionality are shaped by both the individual and the material: "while agency and intentionality may not be properties of things, they are not properties of humans either: they are the properties of material engagement, that is, of the gray zone where brain, body and culture conflate" ([11], p. 22, italics in the original). Moreover, from the perspective of embodied cognition, as well as neuroachaeology, the brain is no longer a mere fixed biological entity, but a "dynamic bio-cultural system subject to constant cultural and structural transformations" ([15], p. 55).

In the case of mathematics education, teacher and students participate constantly in material/tool engagement by manipulating 'things' within the classroom. For example, the form the blackboard is used has deep roots in local education cultures (see [16]). Moreover, mathematical concepts represent objects taking a particular role in the classroom. Such objects, concepts, co-evolve with each student, and with the teacher, forming a part in the process of structural coupling and structural determination. As an example we can consider how every individual, student or teacher, makes sense of, or develops, a mathematical concept or process through problem solving. In the same form that material objects can be used as tools and artifacts in a specific activity, mathematical concepts are intellectual tools empowering individuals to undertake endeavors that might be very challenging, or even not possible, otherwise. For instance, students may use polynomials for modeling and approaching mathematical problems.

The use of different tools when undertaking a learning activity both liberates and constrains the brain and the mind: this isthe agency,and intentionality, within the relationship. In this line of though artifacts, such as computers and electronic tablets, are not only physical matter, but they are also cultural tools that become part of the mind when used in an activity such as solving mathematical problems. From a neuroarchaelogical perspective, the use of objects, new technologies in this case, not only empowers, but also transforms our minds, and our brains, both culturally and physiologically (see [15]).

The interactions among teacher, students, technology, and mathematical tasks within a problem solving learning environment represent a complex system embedded in a cultural context. Technology, such as the iPad in this case, includes the hardware, the functional software or applications, as well as the form that teacher and students use it. The agency enacted in the relationship between student and software determines not only the mathematical activity, but also the form of student and teacher participation in the classroom. The original design purpose of the software shapes the agency and intentionality between the iPad and the student. In the next section I differentiate between two different design purposes of software, and its applications, regarding mathematics education.

\section{SPECIALIZED AND NON-SPECIALIZED SOFTWARE}

For the discussion presented in this paper I distinguished two types of Software used for mathematics education according to its original purpose and common use. For instance, the spreadsheet, such as Excel, has multiple uses outside the school. Although it has been used as a tool for learning algebra and calculus, the use of this software is not limited to learning mathematics. For this reason I referred to it as non-specialized software for mathematics education. However, an Excel file can be specialized for mathematics education as long as it has been designed to a particular learning activity. In contrast, other software is specifically designed for mathematics learning, such as the Geometer's SketchPad. Applications and activities of this type of software are often targeted to some particular mathematical concepts or skills to be developed by students. The specialization of some of these applications has great advantages. Students can master targeted skills and develop particular concepts intentionally. Sometimes an adult is not even necessary for the student to get engaged with the mathematical task offered by the software.

In a less prescriptive context for learning and teaching with the technology, students are free to use the software in different forms. The electronic tablet, for example, offers a number of applications which may serve as tools for mathematics learning, even though these applications have not been designed with that particular purpose of mathematics education. For example, applications including the calculator, spreadsheet, camera, and video and voice recording can be used in the analysis and solutions of a mathematical problem, as well as a means to communicate students' reasoning, represent their ideas and visualize and organize data in different forms. Additionally, by communicating their approaches to the mathematical tasks, students have the potential to contribute to collective learning of the classroom, as a broader unit, in an unexpected fashion.

\section{AN EXAMPLE FROM THE CLASSROOM}

My experience as a teacher educator has focused on the collaborative design of mathematical tasks with teachers and other educators as a means of professional development and curriculum design (e.g. [16]). These tasks were included in lessons in which the technology often played an increasingly important role. Recently, I had the opportunity to work with two teachers from a public high school whose students were assigned an iPad2 as part of their learning tools set. They used this technology at school and were able to take it home, as well.

During one of these collaborative activities we, a team of educators from the Galileo Educational Network Association (GENA) and the two teachers, mathematics and science, in the school planned a unit together for a grade 10 course. We met every week for one hour during a whole academic year. In those meetings the two educators, including myself, collaborated in the design of mathematics and science problems and activities with the teachers who were co-teaching mathematics and science.

The teachers decided to mix the two courses. For most of the year the units of the both courses, mathematics and science, were alternated. In this way, students focused either on mathematics or on science. Having both courses joint in this fashion allowed longer sessions. The desks 
were set in the classroom in groups of four, facilitating collaboration among students.

The use of the iPad2 was not limited to mathematics in the groups of students who were taught by these teachers. The team also designed and participated in a science projects in which students used the tablet for data collection, analysis and representation of their conclusions, including the generation of video. Additionally, other educators from GENA were using this technology for social science projects in which these students used VoiceThread, and iPad application, to create video recording as part of the activities. In this sense, the technology was used widely by these students. However, they used the iPad2 for the first time in the mathematics activity described in this paper. A further discussion about the interactions of this technology among teachers and students in other disciplines is beyond the scope of this article. What is important here is to stress the varied use of the technology by these students. The tablet became a learning tool inside and outside the classroom for different disciplines.

The unit we design together was about algebraic operations among polynomials. In particular, the unit focused on the addition, subtraction and product of polynomial. We wanted to include a rich mathematical problem in which students not only had to propose their own approaches and solutions, but also communicate their reasoning,so most of the mathematics learning processes described by the WNCP would be approached, in this case: communication, connections, problem solving, reasoning, technology, and visualization. We decided to use one rich mathematical problem during the whole unit. Students had to work on this problem for several lessons, including a quiz by the end on the unit. Additionally, short segments of each lesson were used to revisit and expand students' knowledge on the algebraic operations with polynomials. We called these segments the 'mini-lessons,' which also included some practice exercises. The rich mathematical problem (adapted from [18]) is described as follows.

Background Information: A cube's dimensions (l, w, and h) match its "age." In other words, on its first birthday, a cube's dimensions are $1 \times 1 \times 1$. On its second birthday, its dimensions are $2 \times 2 \times 2$. Every year on its birthday, the entire cube is dipped in paint. Only the exposed faces get a paint job.

Focusing Questions: How many blocks would have 0, 1,2 , and 3 painted faces on a 10 -year old cube? On an nyear old cube?

The unit was giving in six sessions, comprising the regular time for mathematics and science. Students spent three class sessions working on the rich problem. In the first session the problem was posed and students worked on it, both individually and by teams, without the aid of the iPad2. Manipulative materials were given to students, including Rubik's cubes and blocks. Students started working on the problem and they began to generate some formulas for the general case of cubes with a $0,1,2$, and 3 painted faces.

In the second session the iPad2 was given to students, who were asked to represent their findings in the application 'Numbers,' which is a spreadsheet. In this lesson, the teacher briefly explained how to introduce formulas in the application using the reference to a cell. She showed how

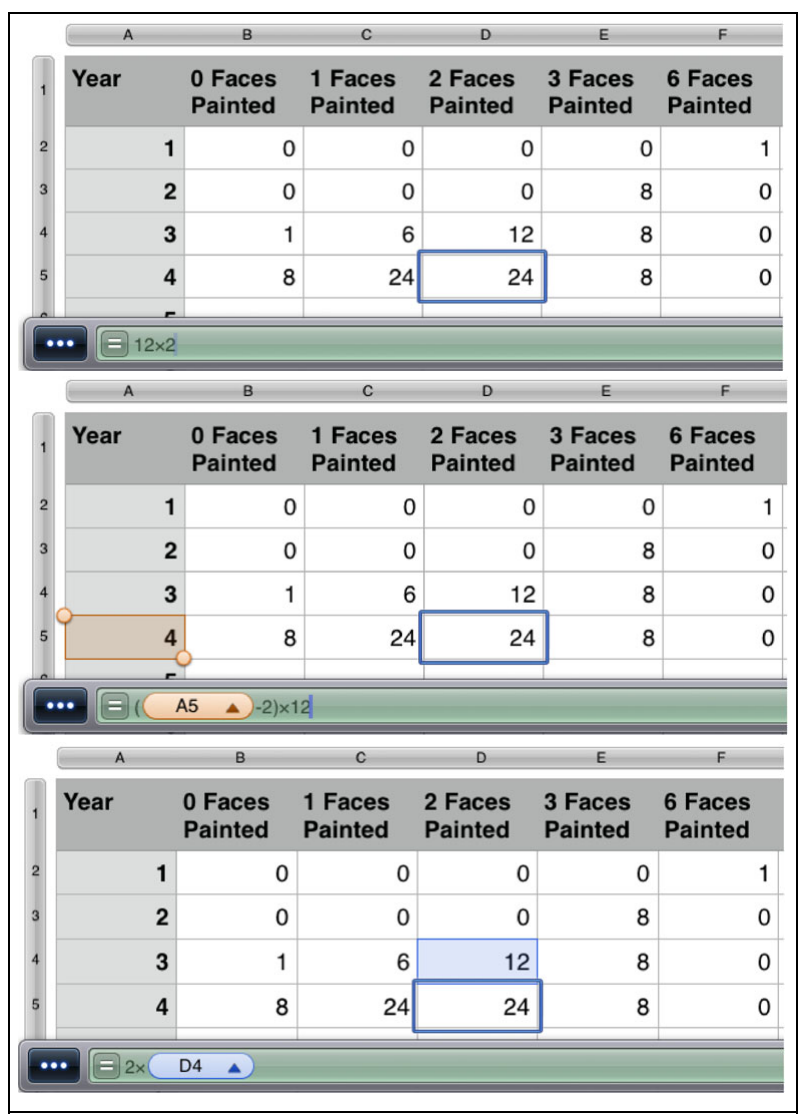

Figure 1. Different uses of the spreadsheet: a) in the top, arithmetic operations, b) in the middle, a recursive formula, and c) in the bottom, a general formula.

to generate the sequence of consecutive numbers in a column that represented the years. Every year, except the first one, was the value of the previous year plus one. Students recorded their findings for some of the numbers of faces painted in each year. They were suggested to include formula for their findings.

I identify fours different students' forms of using of the spreadsheet. The first form was counting the number of painted faces and recording the numbers in the spreadsheet. This form did not required to insert any formula and the application was used only as a chart to record information. The number of cubes in each case was either counted by the students, or calculated using arithmetic operations without this application. The second form was performing arithmetic operations in the spreadsheet making it easier to find the number of painted faces. The third form consisted of introducing a recursive formula, and the forth was the use of a general formula. Some students mixed some of these forms in their initial work as they were discovering different properties and ways of finding the corresponding numbers of painted faces. Looking at students' work in the iPad, it was possible to identify each of these uses of the spreadsheet. For instance, the number of cubes with two faces painted can be obtained by both a recursive and an explicit formula. Examples of the second, third and fourth cases are shown in Fig. 1. The recursive formula is obtained by adding 12 to the number of cubes with two faces painted in the previous year (Fig. 1b). The general formula can be obtained by multiplying by 12 the numbers of years reduced by two (Fig. 1c). 
Being able to identify students' use of the spreadsheet allowed us to have an idea of the type of reasoning of each student. That is, we were able to identify whether a student worked concretely in each case, or generalized with either a recursive or explicit formula. By this lesson students were very familiar with the problem. The agency of the relationship between the mathematical task and the students shifted.

In these two lessons most students were generating algebraic expressions for each numbers of cubes with a determined number of painted faces. Some of them released that these formulas may be expanded. However did not remember how to do this properly (Fig. 2). The spreadsheet was used for verifying the formulas numerically in each case: The volume of the cube in each year, measures in small cubes, must be equal to the sum of all the cubes, as shown in (1).

$$
(n-2)^{3}+6(n-2)^{2}+12(n-2)+8=n^{3}
$$

A review of how to multiply polynomials was given in one of the mini-lessons. A visual representation for the product was explained to the group, and students used the tablet to work on some exercises (Fig. 3). The use of the iPad2 represented some potential advantages: (1) students were able to redo the exercise easily in case of mistake, (2) their work could be saved in the memory of the devise, and (3) it was easy to present students' work to the whole group in the SmartBoard.

Some students used a calculator to perform computations during this session despite the fact that they were using the Numbers application in the tablet. Explanations of how to use the iPad to perform these computations were given. However, one student still preferred to use the calculator, probably because of the familiarity with it. Not being familiar with the Numbers application was a limitation in this case. This limitation exemplifies the agency and intentionality of the relationship between the tool and the student. This limitation might change when the student familiarizes with the application. Once students use the iPad as a tool for mathematics problem solving in new forms, they represent something different to them. That is, the relationship between the student and the iPad changes.

During this second session I noticed that some students showed the teacher how to use certain features of the iPad such as splitting the keyboard when introducing text or numbers. This feature allowed the user to have a broader visual space when typing in the tablet (Fig. 4). Such feature was also new for me and I learned that in order to include it in my tablet, I needed to upgrade the operating system. This feature was discovered by one of the students and soon many people, including us, knew how to split the keyboard. In this case, using this feature of the device facilitates the work, making s shift in the agency between the device and the user, including students, teachers and educators.

During this second lesson students and teacher discussed about the process of solving a problem and the explanation of a solution. From two examples of processes and solutions of a different problem, students and teacher generated two assessment rubrics: the first for the process of solving the problem, and the second for the explanation of the solution. Students used this rubric to self assess their work.
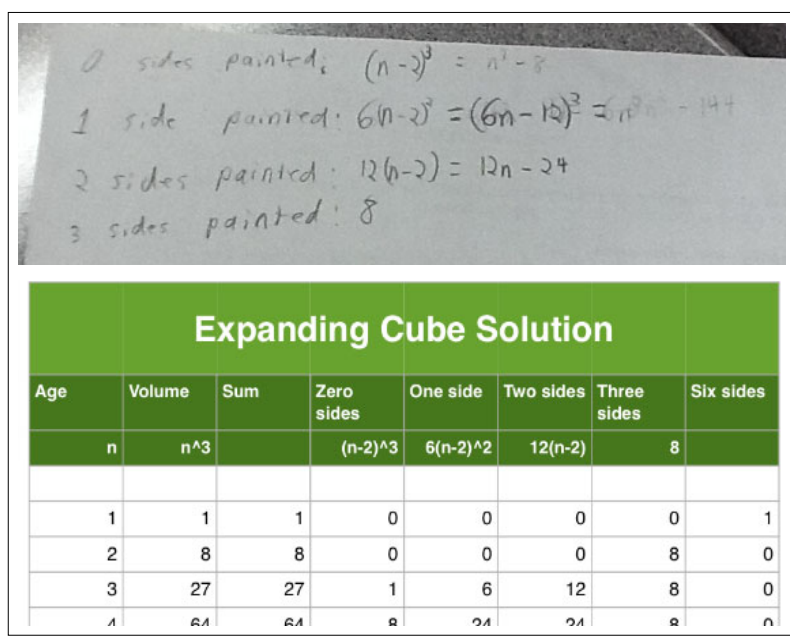

Figure 2. Students used the iPad to verify their formulas. The image on the top shows the formulas developed in paper. In the bottom an example of the use of the spreadsheet is presented.

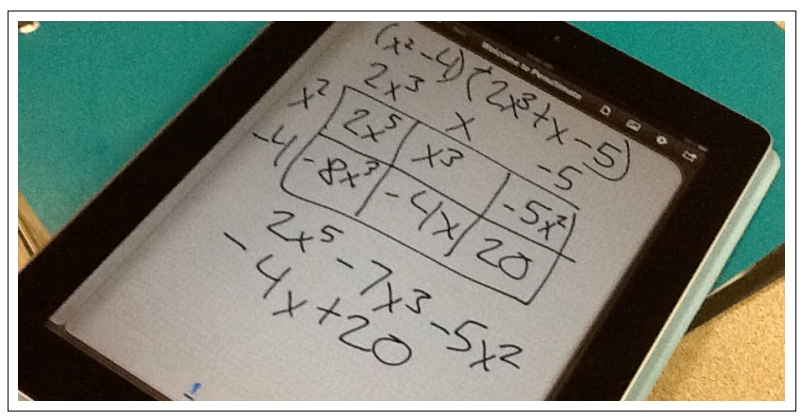

Figure 3. Students used the iPad to work on exercises involving product of polynomials.

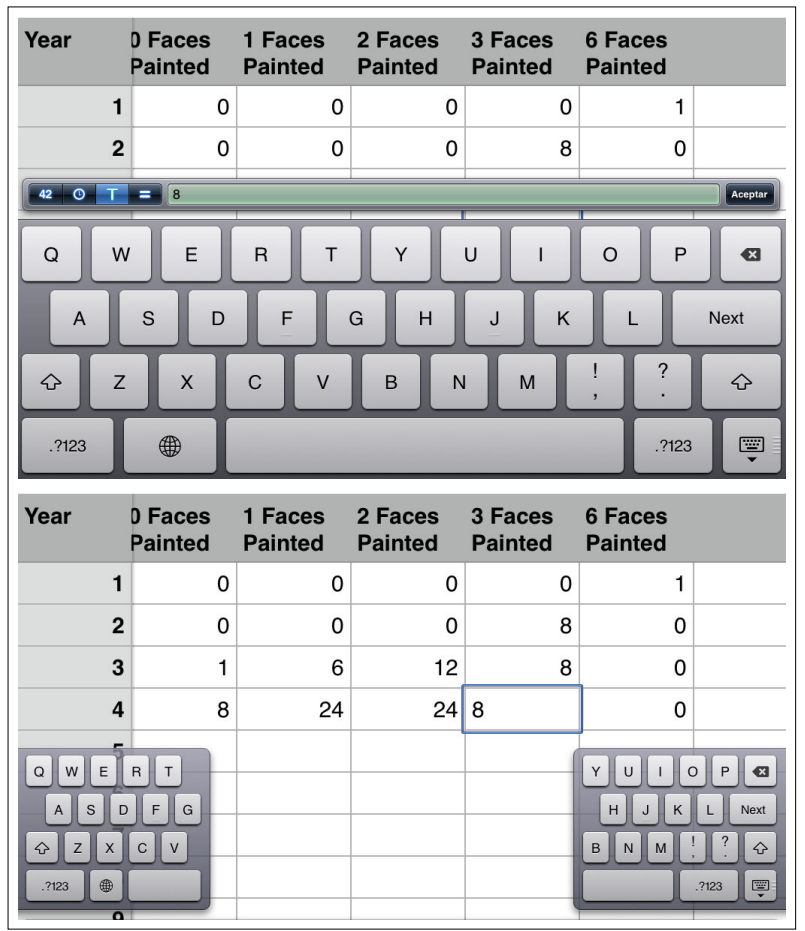

Figure 4. The keyboard of the iPad may cover a big part of the screen, making it difficult to see the part that is being typed (figure on top). However, the keyboard can be split and reduced avoiding to cover a big part of the screen (figure on bottom). 
In the third session the teacher generated, from students' suggestions, an assessment rubric for both the problem solving process and the presentations of the solution of the problem. They were asked to use the iPad2 to present their solution to the problem however they wanted. Some of them used 'Pages,' a word processor for the iPad, to present explanations to their solutions. Edited photographs were added to the presentations as a means to explain how they demonstrated the correct use of the formulas, as shown in Fig. 5. Other students used the video camera of the iPad to present their solutions to the problem. No instruction to the use of these applications was given to the students. They explored and used them by their own. The interaction between the students and the mathematics task, as well as the student and the iPad, evolved during the course of these three lessons.

During this unit the problem was used to assess addition and product of polynomials in a quiz. Students new the formulas for the number of painted cubes in each case (Fig. 2). The test consisted on proving algebraically that the sum of all the expressions was equal to the total number of cubes, as in (1).

\section{CONCLUSIONS}

When different approaches to solve a mathematical task are communicated in the classroom, there is a learning opportunity for both teachers and students. As mentioned before, communication and visualization are also process involved in mathematics learning. In the experience presented here, the use of the iPad2 represented opportunities for the exploration of different approaches to the task, as well as different forms of communication and representation of students' solutions to the problem. These opportunities are fertile terrain for the natural drift of potential learning that can be developed in the classroom by knowledgeable teachers and learners. Such learning is not limited to mathematical formulas, but also includes the use of the technology for data analysis, representation, and visualization, as well as the communication of the student's thinking.

The relationships among mathematical tasks, tools, students, and teacher are complex and culturally situated. The iPad2 is not only a learning tool, but also a common artifact in our technological society. Moreover, it is personalized: the user can configure an iPad2 for his, or her, own specifications, with or without the context of school. The interaction between the iPad2 and the student may indeed be more intimate than it is with other artifacts that are used only for the learning of targeted mathematics tasks, such as the calculator. The notion of agency and intentionality can be used to help us grasp new insights on the interaction of technology with the student, particularly when the students (and teachers) are engaged in mathematics problem solving.

Students created knowledge in the classroom in more than one sense. In addition to the mathematical knowledge developed in the classroom, students learnt that they can contribute to collective learning also. This is a position or stance on teaching and learning that goes well beyond the repetition of procedures and perpetuation of knowledge, and it is integrated with the sensibility that tools and tasks shape, and are shaped by, learning and learning collectives as sets of relationships. Although this paper contains an example of the use of the electronic tablet in the class

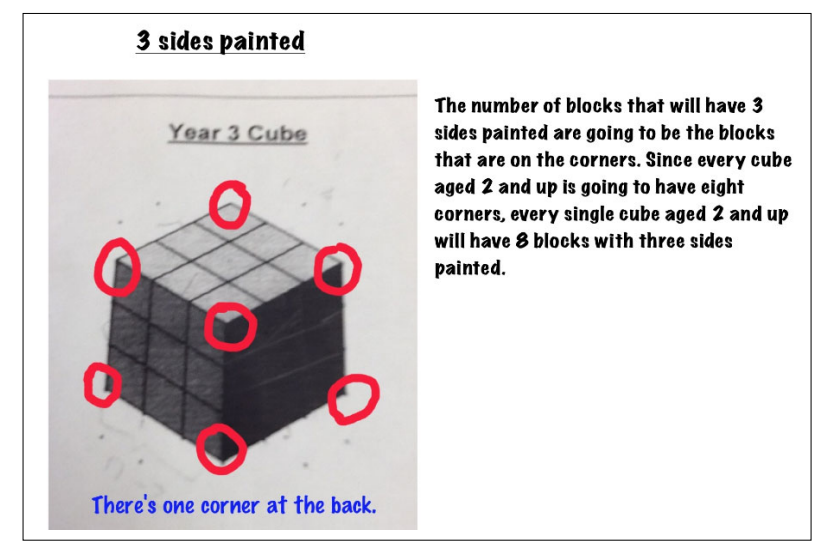

Figure 5. Sample of student presentation of a part of the solution of the problem.

room, the features of this device for media manipulation and virtual communication are barely included. 'Digital Native' studentsare not onlyknowledgeable of how to use particular software, but also participate in broader virtual communities and study, work and interact in new forms [8]. We, educators, must acknowledge such abilities and reconsider the roles of, and interactions among, students, teacher, technology and mathematics in the classroom. The perspective provided in this article serves as a basis to conceptualize such roles and interactions for both educators and researchers.

\section{REFERENCES}

[1] A. Gutiérrez, and P. Boero, Eds., "Handbook of research on the Psychology of Mathematics Education: Past present and Future.” Rotterdam, The Netherlands: Sense Publishers, 2006.

[2] WNCP, "The common curriculum framework for grades 10 to 12 mathematics.” WNCP, 2008.

[3] WNCP, "The common curriculum framework for grades K to 9 mathematics." WNCP, 2006. Retrieved February, 2012 from: http://www.wncp.ca/english/subjectarea/mathematics/ccf.aspx

[4] NCTM, “The Learning Principle.” NCTM, 2012. Retrieved February, 2012 from: http://www.nctm.org/standards/content.aspx ?id=2680

[5] I. Handlovská, and T. Kozík. "The reduction of interest among elementary students in the field of technical education" in iJEP vol. 1(3), 2011, pp. 9-12. Retrieved April, 2012 from http://dx.doi.org/ijep.v1i3.1822

[6] R. Leikin\& R. Zazkis, Eds., "Learning through teaching mathematics: Development of teachers' knowledge and expertise in practice.” Springer, 2010. http://dx.doi.org/10.1007/978-90-4813990-3

[7] A. B. Anthony, and M. L. Clark. "Examining dilemmas of practice associated with the integration of technology into mathematics classrooms serving urban students." Urban Education, vol. 46 (6), 2011, pp. 1300-1331. ERIC. Web. 29 Jan. 2012.

[8] J. Palfrey and U. Gasser, "Born Digital: Understanding the First Generation of Digital Native.” New York: Basic Books, 2008.

[9] C. Burwell, "Rewriting the script: Towards a politics of young people's digital media participation.” The Review of Education, Pedagogy and Cultural Studies, vol. 32(5), 2010, p. 382-402. http://dx.doi.org/10.1080/10714413.2010.510354

[10] H. R. Maturana, and F. J. Varela, "The three of knowledge” Rev. ed. Boston: Shambhala, 1992.

[11] L. Malafouris, “At the potter's wheel: An argument for material agency” in Material agency: Towards a non-anthropocentric approach, C. Knappett ad L. Malafouris, Eds. New York: Springer, 2008, pp. 19-36.

[12] B. Davis, and D. Sumara. “Complexity and Education.” Lawrence Erlbaum Associates: Mahwah, NJ, 2006. 
PAPER

INCORPORATING THE IPAD2 IN THE MATHEMATICS CLASSROOM: EXTENDING THE Mind INTO THE COLLECTIVE

[13] J. Proulx, E. Simmt, and J. Towers, "The enactivist theory of cognition and mathematics education research: Issues of the past, current questions and future directions” in Proceedings of the 33rd of the International Group for the Psychology of Mathematics Education, Vol. 1, M. Tzekaki, M. Kaldrimidou, and H. Sakonidis, Eds. Thessaloniki, Greece. 2009, pp. 249-278.

[14] M. Soliman and C. Gütl, "Evaluation of Intelligent Agent Frameworks for Human Learning in Virtual Worlds" in iJEP vol. 1 (3), 2011, pp 45-48.

[15] L. Malafouris, "Metaplasticity and the human becoming: Principles of neuroarchaeology.” Journal of Anthropological Sciences, vol. 88, 2010, pp. 49-72.

[16] J. Stigler \& J. Hiebert, The teaching gap: Best ideas for the world's teachers for improving education in the classroom. New York: The Free Press, 1999.

[17] A. P. Preciado-Babb, and P. Liljedahl. "Three Cases of Teachers' Collaborative Design: Perspectives From Those Involved. Canadian” in Journal of Science, Mathematics and Technology Educa- tion, vol 12(1), 2012, pp 22-35. http://dx.doi.org/10.1080/149261 $\underline{56.2012 .649052}$

[18] Thinking mathematically, April/May 2006. Retrieved November 2011 from: www.nhadulted.org/thinkingmathematically/Issue8_05 06.pdf

\section{AUTHOR}

A. P. Preciado-Babb is a postdoctoral fellow at the Galileo Educational Network Association and the Faculty of Education of the University of Calgary, Calgary, AB, Canada (e-mail: apprecia@ucalgary.ca).

This work was supported by Gallager-Galileo Fellowship and SSHRC, Canada 410-2009-0347. It is an extended version of a paper presented at the International Conference IEEE EDUCON2012, held in April 2012, in Marrakesh, Morocco. Received, 22 April 2012. Published as resubmitted by the author 30 April 2012. 